\title{
THE AXISYMMETRIC RESPONSE OF A FLUID-FILLED SPHERICAL SHELL TO A LOCAL RADIAL IMPULSE-A MODEL FOR HEAD INJURY*†
}

\author{
ALI E. ENGIN \\ Highway Safety Research Institute, The University of Michigan, Ann Arbor, Mich. 48107, U.S.A.
}

\begin{abstract}
This investigation is concerned with determination of the dynamic response of a fluid-filled spherical shell subjected to a local radial impulsive load. From the application point of view, such a fluid-shell system is considered to be a simple, but to date, the most improved theoretical model representing the human head when subjected to impulsive external loads. Analysis is based on linear shell theory which includes both membrane and bending effects. The motion of the fluid is assumed to be governed by the wave equation. Laplace transform technique is used in obtaining the transient axisymmetric response of the system to a local radial impulsive load. The solutions thus obtained for the velocity potential of the fluid and the displacement components of the shell mid-surface are the Green's functions of the problem with respect to time. Some numerical results for the theoretical model are obtained for a set of appropriate data. The comparison is made for the stress distributions at various times in the shell for both the empty and the fluid-filled cases. In the fluid-filled case the excess pressure propagation in the fluid is also discussed. The possible locations of brain damage and skull injury are indicated on the basis of the numerical computations.
\end{abstract}

\section{INTRODUCTION}

THE SUBJECT matter of this investigation received its stimulus from the following two considerations. First of all, the complete determination of the dynamic response of a fluid-filled shell subjected to a local radial impulsive load is a point of interest in theoretical mechanics due to the fluid-solid interaction nature of the problem. Secondly, it is hoped that a fluid-filled spherical shell will serve as a simple but improved theoretical model representing the human head when subjected to impulsive external loads.

As it was pointed out by Goldsmith (1966), the various mechanisms of skull and brain damage proposed by previous investigators are of little practical value in quantitative determination of the location and magnitude of brain trauma and head injury. In the literature there are no rigorous mathematical treatments of the theoretical head injury models except for the fluid-filled rigid shell model analyzed by Anzelius (1943) and
Guittinger (1950). Their formulation are essentially identical and involve an axisymmetric solution of the wave equation in spherical coordinates. In the papers of both authors the eigen values of the problem are determined by requiring the radial component of the fluid velocity to vanish at the interior surface of the rigid spherical shell surrounding the fluid. In the analysis of Anzelius, the spherical vessel containing the fluid has constant translational velocity for $t<0$. At $t=0$ the vessel is brought to a sudden stop. From this physical situation he gets one initial condition for the velocity potential, $\Phi$, of the fluid and assumes the initial pressure distribution which supplies the second initial condition on the time derivative of $\Phi$. In Güttinger's analysis the fluid-filled spherical vessel is initially at rest. At $t=0 \mathrm{a}$ 'momentary impact' force instantaneously accelerates the vessel to a constant velocity which the vessel retains for all $t>0$. Again from the physical situation the first initial condition on $\Phi$ can

*Received 22 November 1968.

$\uparrow$ Presented at the ASME Third Biomechanical and Human Factors Division Conference at the University of Michigan, Ann Arbor, June 12-13, 1969. 
be written down immediately and the second initial condition on the time derivative of $\Phi$ is equal to zero since the initial dynamic pressure distribution in this case is zero. Both authors concluded that an initial compression wave arises from the pole of impact, and due to the rigidity of the shell, instantaneously a tension (rarefaction) wave is emitted from the counterpole, both traveling towards the geometric center of the system. The superposition of the two waves at the center produces large changes in the fluid pressure and this phenomena was considered to be the cause of brain trauma. It should be remarked that the assumption of rigidity of the shell causes an infinite speed of wave propagation in the container and, as a direct consequence of this, every point of the interior surface of the container instantaneously becomes a source of varying strength which transmits energy into the fluid. The obvious shortcomings of the fluid-filled rigid shell model led Goldsmith to suggest the construction of a fluid-filled elastic shell model and its analytical or numerical solution. Goldsmith's paper was primarily addressed to those who are outside of the discipline of mechanics; however, a thorough review of previously employed theoretical and experimental methods describing the formation of brain trauma and head injury has been given.

The theoretical model for the present investigation consists of a thin elastic spherical shell filled with inviscid compressible fluid. The shell material and fluid are considered to be homogeneous and isotropic. The loading pattern is to be taken local, radial, impulsive and axisymmetric. Since the load is applied locally the combined linear shell theory which includes membrane and bending effects of the shell is used for the proper description of the wave propagation.

\section{EQUATIONS OF MOTION AND THEIR TRANSFORMS}

This author (1968) obtained the governing differential equations of a fluid-filled spherical shell by means of Hamilton's Principle. These three partial differential equations which are coupled in terms of the tangential and radial displacements $u, w$ of the shell mid-surface and velocity potential, $\Phi$, of the fluid are given below in nondimensional form:

$$
\begin{aligned}
& \alpha^{2}\left[\frac{\partial^{2} \psi}{\partial \varphi^{2}}+\cot \varphi \frac{\partial \psi}{\partial \varphi}-\left(\nu+\cot ^{2} \varphi\right) \psi-\frac{\partial^{3} \zeta}{\partial \varphi^{3}}\right. \\
& \left.-\cot \varphi \frac{\partial^{2} \zeta}{\partial \varphi^{2}}+\left(\nu+\cot ^{2} \varphi\right) \frac{\partial \zeta}{\partial \varphi}\right]+\frac{\partial^{2} \psi}{\partial \varphi^{2}}+\cot \varphi \frac{\partial \psi}{\partial \varphi} \\
& -\left(\nu+\cot ^{2} \varphi\right) \psi+(1+\nu) \frac{\partial \zeta}{\partial \varphi}-\frac{\partial^{2} \psi}{\partial \tau^{2}}=0 \\
& \alpha^{2}\left[\frac{\partial^{3} \psi}{\partial \varphi^{3}}+2 \cot \varphi \frac{\partial^{2} \psi}{\partial \varphi^{2}}-\left(1+\nu+\cot ^{2} \varphi\right) \frac{\partial \psi}{\partial \varphi}\right. \\
& +\cot \varphi\left(2-\nu+\cot ^{2} \varphi\right) \psi-\frac{\partial^{4} \zeta}{\partial \varphi^{4}}-2 \cot \varphi \frac{\partial^{3} \zeta}{\partial \varphi^{3}} \\
& \left.+\left(1+\nu+\cot ^{2} \varphi\right) \frac{\partial^{2} \zeta}{\partial \varphi^{2}}-\cot \varphi\left(2-\nu+\cot ^{2} \varphi\right) \frac{\partial \zeta}{\partial \varphi}\right] \\
& -(1+\nu)\left(\frac{\partial \psi}{\partial \varphi}+\cot \varphi \psi+2 \zeta\right)-\frac{\partial^{2} \zeta}{\partial \tau^{2}} \\
& -f \frac{\partial \Phi_{1}(1, \varphi, \tau)}{\partial \tau}=-\frac{\left(1-\nu^{2}\right)}{E h} a F(\varphi) \delta(\tau)
\end{aligned}
$$

$$
\begin{array}{r}
\frac{1}{r_{1}^{2}} \frac{\partial}{\partial r_{1}}\left(r_{1}^{2} \frac{\partial \Phi_{1}}{\partial r_{1}}\right)+\frac{1}{r_{1}^{2} \sin \varphi} \frac{\partial}{\partial \varphi}\left(\sin \varphi \frac{\partial \Phi_{1}}{\partial \varphi}\right) \\
-\frac{1}{s^{2}} \frac{\partial^{2} \Phi_{1}}{\partial \tau^{2}}=0
\end{array}
$$

where

$$
\begin{aligned}
& \psi=\frac{u}{a}, \quad \zeta=\frac{w}{a}, \quad \tau=\frac{c_{s}}{a} t, \quad c_{s}=\left[\frac{E}{\rho_{s}\left(1-\nu^{2}\right)}\right]^{1 / 2} \\
& s=\frac{c}{c_{s}}, \quad r_{1}=\frac{r}{a}, \quad \Phi_{1}=\frac{\Phi}{a c_{s}}, \quad f=\frac{\rho_{0} a}{\rho_{s} h}
\end{aligned}
$$

The external load, designated by $F_{e}=$ $F(\varphi) \delta(\tau)$ in equation (2), is assumed to be axisymmetric and its impulsive nature is expressed by means of the Dirac delta function, $\delta(\tau)$, the properties of which are accepted here in the usual sense seen in applied mathematics. When the external load, $F_{e}$, is applied 
to the fluid-filled shell which is initially at rest relative to the inertial reference the mass center of the sphere will experience a rigid body velocity, $V_{c}$, with a step function $H(t)$ behavior due to the impulsive nature of the external load. The value of this velocity is:

$$
V_{c}=\frac{H(t)}{M} \int_{S} F(\varphi) \mathrm{d} S,
$$

where

$$
\begin{aligned}
M= & m_{s}+m_{0} \text { is the total mass of the } \\
& \text { system } \\
F(\varphi)= & \text { external load intensity per unit } \\
& \text { mid-surface area of the shell } \\
\mathrm{d} S= & 2 \pi a^{2} \sin \varphi \mathrm{d} \varphi .
\end{aligned}
$$

Since the fluid-filled shell is assumed to be at rest prior to the application of the radial impulsive load, all the initial conditions relevant to the differential equations (1), (2) and (3) are homogeneous, i.e.,

$$
\begin{array}{ll}
1 \zeta(\varphi, 0)=0, & 2 \frac{\partial \zeta(\varphi, 0)}{\partial \tau}=0, \\
3 \psi(\varphi, 0)=0, & 4 \frac{\partial \psi(\varphi, 0)}{\partial \tau}=0, \\
5 \Phi_{1}\left(r_{1}, \varphi, 0\right)=0, & 6 \frac{\partial \Phi_{1}\left(r_{1}, \varphi, 0\right)}{\partial \tau}=0 .
\end{array}
$$

Let the following to be the notation for the Laplace transform of a function $F(\tau)$ with respect to $\tau$

$$
L\{F(\tau)\}=\bar{F}(p)=\int_{0}^{\infty} \mathrm{e}^{-p \tau} F(\tau) \mathrm{d} \tau,
$$

where $p$ is a complex variable. Since $\zeta, \psi$, and $\Phi_{1}$ are functions of more than one independent variable, let their Laplace transforms be denoted by

$$
\begin{aligned}
L_{\tau}\{\zeta(\varphi, \tau)\} & =\bar{\zeta}(\varphi, p)=\bar{\zeta}, \\
L_{\tau}\{\psi(\varphi, \tau)\} & =\bar{\psi}(\varphi, p)=\bar{\psi}, \\
L_{\tau}\left\{\Phi_{1}\left(r_{1}, \varphi, \tau\right)\right\} & =\bar{\Phi}_{1}\left(r_{1}, \varphi, p\right)=\bar{\Phi}_{1} .
\end{aligned}
$$

Using the notation defined in (6) and the initial conditions (5) the Laplace transforms of equations (1), (2) and (3) with respect to the nondimensional time, $\tau$, are:

$$
\begin{aligned}
& \alpha^{2}\left[\frac{\mathrm{d}^{2} \bar{\psi}}{\mathrm{d} \varphi^{2}}+\cot \varphi \frac{\mathrm{d} \bar{\psi}}{\mathrm{d} \varphi}-\left(\nu+\cot ^{2} \varphi\right) \bar{\psi}-\frac{\mathrm{d}^{3} \bar{\zeta}}{\mathrm{d} \varphi^{3}}\right. \\
& \left.-\cot \varphi \frac{\mathrm{d}^{2} \bar{\zeta}}{\mathrm{d} \varphi^{2}}+\left(\nu+\cot ^{2} \varphi\right) \frac{\mathrm{d} \bar{\zeta}}{\mathrm{d} \varphi}\right]+\frac{\mathrm{d}^{2} \bar{\psi}}{\mathrm{d} \varphi^{2}}+\cot \varphi \frac{\mathrm{d} \bar{\psi}}{\mathrm{d} \varphi} \\
& -\left(\nu+\cot ^{2} \varphi\right) \bar{\psi}+(1+\nu) \frac{\mathrm{d} \bar{\zeta}}{\mathrm{d} \varphi}-p^{2} \bar{\Psi}=0,
\end{aligned}
$$

$$
\begin{aligned}
& \alpha^{2}\left[\frac{\mathrm{d}^{3} \bar{\psi}}{\mathrm{d} \varphi^{3}}+2 \cot \varphi \frac{\mathrm{d}^{2} \bar{\psi}}{\mathrm{d} \varphi^{2}}-\left(1+\nu+\cot ^{2} \varphi\right) \frac{\mathrm{d} \bar{\psi}}{\mathrm{d} \varphi}\right. \\
& +\cot \varphi\left(2-\nu+\cot ^{2} \varphi\right) \bar{\psi}-\frac{\mathrm{d}^{4} \bar{\zeta}}{\mathrm{d} \varphi^{4}}-2 \cot \varphi \frac{\mathrm{d}^{3} \bar{\zeta}}{\mathrm{d} \varphi^{3}}
\end{aligned}
$$

$\left.+\left(1+\nu+\cot ^{2} \varphi\right) \frac{d^{2} \bar{\zeta}}{d \varphi^{2}}-\cot \varphi\left(2-\nu+\cot ^{2} \varphi\right) \frac{d \bar{\zeta}}{d \varphi}\right]$ $-(1+\nu)\left(\frac{\mathrm{d} \bar{\psi}}{\mathrm{d} \varphi}+\cot \varphi \psi+2 \bar{\zeta}\right)-p^{2} \bar{\zeta}$

$-p f \Phi_{1}(1, \varphi, p)=-\frac{\left(1-\nu^{2}\right) a F(\varphi)}{E h}$

$\frac{1}{r_{1}^{2}} \frac{\partial}{\partial r_{1}}\left(r_{1}{ }^{2} \frac{\partial \bar{\Phi}_{1}}{\partial r_{1}}\right)+\frac{1}{r_{1}^{2} \sin \varphi} \frac{\partial}{\partial \varphi}\left(\sin \varphi \frac{\partial \bar{\Phi}_{1}}{\partial \varphi}\right)-\frac{p^{2}}{s^{2}}$

$$
\bar{\Phi}_{1}=0 \text {. }
$$

The transform of the boundary condition between fluid and shell is:

$$
p \bar{\zeta}(\varphi, p)=\frac{\partial \bar{\Phi}_{1}(1, \varphi, p)}{\partial r_{1}} .
$$

Thus, the problem has been reduced from the solution of three partial differential equations to that of one partial differential equation and two ordinary differential equations in the transform space.

Before proceeding with the solution of the above equations, we first expand the function

$$
g(\varphi)=\left\{\begin{array}{l}
F(\varphi), 0<\varphi<\varphi_{0} \\
0, \varphi_{0}<\varphi<\pi
\end{array}\right.
$$

in a series of Legendre polynomials of the form

$$
g(\varphi)=\sum_{n=0}^{\infty} F_{n} P_{n}(\cos \varphi) .
$$


In particular, if $F(\varphi)=F=$ constant, then, the coefficients $F_{n}$ are found, by the usual methods, to be

$$
\begin{aligned}
F_{n}=\frac{1}{2} F\left[P_{n-1}\left(\cos \varphi_{0}\right)-P_{n+1}\left(\cos \varphi_{0}\right)\right], & \\
n & =0,1,2, \ldots
\end{aligned}
$$

it being realized of course, that $P_{-1}\left(\cos \varphi_{0}\right) \equiv$ 1.

Next, the method of separation of variables is applied to the partial differential equation (9) to obtain two ordinary differential equations that $R\left(r_{1}\right)$ and $G(\varphi)$ must satisfy. When the assumed solution, $\Phi_{1}\left(r_{1}, \varphi\right)=R\left(r_{1}\right) G(\varphi)$ is substituted into equation (9) the following equations are obtained:

$$
\begin{aligned}
& G^{\prime \prime}(\varphi)+\cot \varphi G^{\prime}(\varphi)+n(n+1) G(\varphi)=0,(12) \\
& R^{\prime \prime}\left(r_{1}\right)+\frac{2}{r_{1}} R^{\prime}\left(r_{1}\right)+\left[k^{2}-\frac{n(n+1)}{r_{1}^{2}}\right] R\left(r_{1}\right)=0
\end{aligned}
$$

where (') denotes differentiation with respect to the argument, $k$ is complex and its value is $\mathrm{pi} / \mathrm{s}$. The bounded solutions of (12) and (13) in the spherical region under consideration are $G(\varphi)=c_{1} P_{n}(\cos \varphi)$ and $R\left(r_{1}\right)=c_{2} j_{n}\left((i p / s) r_{1}\right)$ respectively; where $c_{1}$ and $c_{2}$ are two arbitrary constants. Since the equation (9) is linear, by superposition one can arrive at a formal solution

$$
\bar{\Phi}_{1}\left(r_{1}, \varphi, p\right)=\sum_{n=0}^{\infty} c_{n}(p) j_{n}\left(\frac{i p}{s} r_{1}\right) P_{n}(\cos \varphi),
$$

where the coefficients $c_{n}(p)$ will be determined later. Now, let us consider the two ordinary differential equations (7) and (8). In order to reduce these equations to a pair of equivalent algebraic equations in the 'transform space' assume the following expansions for $\bar{\zeta}$ and $\bar{\psi}$ :

$$
\begin{aligned}
& \bar{\zeta}(\varphi, p)=\sum_{n=0}^{\infty} \bar{a}_{n}(p) P_{n}(\cos \varphi), \\
& \bar{\psi}(\varphi, p)=\sum_{n=1}^{\infty} \bar{b}_{n}(p) \dot{P}_{n}(\cos \varphi) .
\end{aligned}
$$

Substitution of (14) and the first expression of (15) into the transformed boundary condition (10) yields the coefficients $c_{n}(p)$. These coefficients, for each integer value of $n$, are

$$
c_{n}(p)=\frac{\bar{a}_{n}(p)}{\frac{i}{s} j_{n}^{\prime}\left(\frac{i p}{s}\right)} \quad n=0,1,2 \ldots
$$

Thus, the unknown coefficients, $c_{n}(p)$, of the transformed velocity potential, $\bar{\Phi}_{1}\left(r_{1}, \varphi, p\right)$, are expressed in terms of the coefficients, $a_{n}(p)$, of the radial displacement of the shell midsurface in the transform space. Hence (14) can now be written as

$$
\bar{\Phi}_{1}\left(r_{1}, \varphi, p\right)=\sum_{n=0}^{\infty} \frac{\bar{a}_{n}(p)}{\frac{i}{s} j_{n}^{\prime}\left(\frac{i p}{s}\right)} j_{n}\left(\frac{i p}{s} r_{1}\right) P_{n}(\cos \varphi) .
$$

The reduction of the two ordinary differential equations (7) and (8) to algebraic equations in the transform space is accomplished by substituting (11), (15), and (17) into (7) and (8). The equations resulting from the substitutions, contain higher order derivatives of both Legendre and Associated Legendre polynomials. All these derivatives are eliminated by making repeated use of the differential equations satisfied by $P_{n}$ and $P_{n}^{\prime}$. Hence, after some manipulations one obtains the following equations that $\bar{a}_{n}(p)$ and $\bar{b}_{n}(p)$ must satisfy:

For $n=0$

$$
\begin{array}{r}
\left\{p^{2}\left[1+f \frac{1}{\frac{i p}{s}} \frac{j_{0}\left(\frac{i p}{s}\right)}{j_{0}^{\prime}\left(\frac{i p}{s}\right)}\right]+2(1+\nu)\right\} \bar{a}_{0}(p) \\
=\frac{\left(1-\nu^{2}\right) a}{E h} F_{0}
\end{array}
$$

For $n \geqslant 1$

$$
\begin{aligned}
\left\{p^{2}\left[1+f \frac{1}{\frac{i p}{s}} \frac{j_{n}\left(\frac{i p}{s}\right)}{j_{n}^{\prime}\left(\frac{i p}{s}\right)}\right]+p_{2 n}\right\} & \bar{a}_{n}(p)+q_{2 n} \bar{b}_{n}(p) \\
& =\frac{\left(1-\nu^{2}\right) a}{E h} F_{n},
\end{aligned}
$$




$$
p_{1 n} \bar{a}_{n}(p)+\left(p^{2}+q_{1 n}\right) \bar{b}_{n}(p)=0,
$$

where

$$
\begin{aligned}
& p_{1 n}=-(1+\nu)+\alpha^{2}\left(1-\nu-\lambda_{n}\right), \\
& q_{1 n}=-\left(1+\alpha^{2}\right)\left(1-\nu-\lambda_{n}\right), \\
& p_{2 n}=2(1+\nu)+\alpha^{2}\left[\lambda_{n}{ }^{2}-\lambda_{n}(1-\nu)\right], \\
& q_{2 n}=-(1+\nu) \lambda_{n}-\alpha^{2}\left[\lambda_{n}{ }^{2}-\lambda_{n}(1-\nu)\right] .
\end{aligned}
$$

From (18)

$$
\bar{a}_{0}(p)=\frac{R_{0}}{p^{2}\left[1+f \frac{1}{i p} \frac{j_{0}\left(\frac{i p}{s}\right)}{s} j_{0}^{\prime}\left(\frac{i p}{s}\right)\right]+2(1+\nu)},
$$

where

$$
R_{0}=\frac{\left(1-\nu^{2}\right) a F_{0}}{E h}=\frac{\left(1-\nu^{2}\right) a F}{2 E h}\left(1-\cos \varphi_{0}\right) .
$$

Applying Cramer's rule to (19) and (20) we obtain the following expression for $\bar{a}_{n}(p)$ and $\bar{b}_{n}(p)$ for $\geqslant 1$,

$$
\begin{aligned}
& \bar{a}_{n}(p)=\frac{R_{n}\left(p^{2}+q_{1 n}\right)}{\Delta_{n}(p)}, \\
& \bar{b}_{n}(p)=\frac{-\mathbf{R}_{n} p_{1 n}}{\Delta_{n}(p)}
\end{aligned}
$$

where

$$
\begin{aligned}
& R_{n}=\frac{\left(1-\nu^{2}\right) a F_{n}}{E h}=\frac{\left(1-\nu^{2}\right) a F}{2 E h} \\
& {\left[P_{n-1}\left(\cos \varphi_{0}\right)-P_{n+1}\left(\cos \varphi_{0}\right)\right] \text {, }} \\
& \Delta_{n}(p)=\left[1+f \frac{1}{\frac{i p}{s}} \frac{j_{n}\left(\frac{i p}{s}\right)}{j_{n}^{\prime}\left(\frac{i p}{s}\right)}\right] p^{4}+\left\{\left[1+f \frac{1}{\frac{i p}{s}} \frac{j_{n}\left(\frac{i p}{s}\right)}{j_{n}^{\prime}\left(\frac{i p}{s}\right)}\right]\right. \\
& \left.q_{1 n}+p_{2 n}\right\} p^{2}+\left(q_{1 n} p_{2 n}-p_{1 n} q_{2 n}\right) \text {. }
\end{aligned}
$$

Substitution of (22), (23) and (24) into (15) gives the final form of the transformed displacement components of the mid-surface.
These are

$$
\begin{aligned}
& \bar{\zeta}(\varphi, p)= \frac{R_{0}}{p^{2}\left[1+f \frac{1}{i p} \frac{j_{0}\left(\frac{i p}{s}\right)}{\frac{i p}{s} j_{0}^{\prime}\left(\frac{i p}{s}\right)}\right]+2(1+\nu)} \\
&+\sum_{n=1}^{\infty} \frac{R_{n}\left(p^{2}+q_{1 n}\right)}{\Delta_{n}(p)} P_{n}(\cos \varphi), \\
& \bar{\psi}(\varphi, p)=\sum_{n=1}^{\infty} \frac{-R_{n} p_{1 n}}{\Delta_{n}(p)} \dot{P}_{n}(\cos \varphi) .
\end{aligned}
$$

3. INVERSION OF $\bar{\zeta}(\varphi, p), \bar{\psi}(\varphi, p)$, AND $\bar{\Phi}_{i}\left(r_{1}, \varphi, p\right)$ Due to the physical nature of the problem $\zeta, \psi$, and $\Phi_{1}$ should satisfy the following conditions:

(1) $\zeta(\varphi, \tau), \psi(\varphi, \tau)$, and $\phi_{1}\left(r_{1}, \varphi, \tau\right)$ are defined for $\tau \geqslant 0$ and they are each $0\left(\mathrm{e}_{0}^{c}{ }_{0}\right)$, where $c_{0}$ is a constant.

(2) $\zeta(\varphi, \tau), \psi(\varphi, \tau), \Phi_{1}\left(r_{1}, \varphi, \tau\right)$ and their time derivatives are sectionally continuous. Then

$$
\begin{aligned}
& \zeta(\varphi, \tau)=\frac{1}{2 \pi i} \lim _{R \rightarrow \infty} \int_{c-i R}^{c+i R} \\
&\left\{\begin{array}{l}
p^{2}\left[1+f \frac{1}{i p} \frac{j_{0}}{\frac{j_{0}}{s} j_{0}^{\prime}\left(\frac{i p}{s}\right)}\right]+2(1+\nu) \\
s
\end{array}\right] \\
&\left.+\sum_{n=1}^{\infty} \frac{R_{n}\left(p^{2}+q_{1 n}\right)}{\Delta_{n}(p)} P_{n}(\cos \varphi)\right\} \mathrm{e}^{p \tau} \mathrm{d} p,
\end{aligned}
$$


$\psi(\varphi, \tau)$

$$
=\frac{1}{2 \pi i} \lim _{R \rightarrow \infty} \int_{c-i R}^{c+i R}\left\{\sum_{n=1}^{\infty} \frac{-R_{n} p_{1 n}}{\Delta_{n}(p)} \dot{P}_{n}(\cos \varphi)\right\} \mathrm{e}^{p \tau} \mathrm{d} p,
$$$$
\Phi_{1}\left(r_{1}, \varphi, \tau\right)=\frac{1}{2 \pi i} \lim _{R \rightarrow \infty} \int_{c-i R}^{c+i R}
$$$$
\left\{\frac{R_{0} j_{0}\left(\frac{i p}{s} r_{1}\right)}{\frac{i}{s} j_{0}^{\prime}\left(\frac{i p}{s}\right)\left\langle p^{2}\left[1+f \frac{1}{i p} \frac{j_{0}\left(\frac{i p}{s}\right)}{s} j_{0}^{\prime}\left(\frac{i p}{s}\right)\right]+2(1+\nu)\right\rangle}\right.
$$

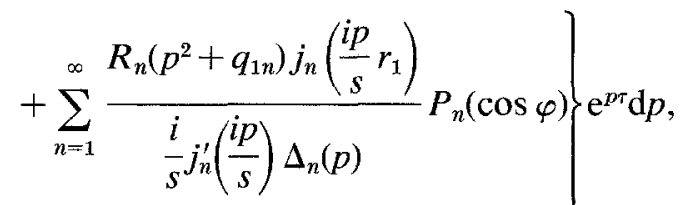

where the path of integration is the line $R e$ $p=c$ in the complex $p$-plane and $c$ is any constant greater than $c_{0}$. As a consequence of a theorem in the theory of complex variables, the functions inside of the braces in (28), (29) and (30) are analytic functions in the half-plane $\operatorname{Re}(p)>c$, i.e., they have no singularities to the right of the line $\operatorname{Re} p=c$. This fact enables us to evaluate the integrals in (28), (29) and (30) by enclosing all the singularities to the left of the line $\operatorname{Re} p=c$ by a suitable contour shown in Fig. 1 and making use of Cauchy's residue theorem. Let any one of the terms inside of the braces in (28), (29), and (29), and (30) be denoted by $f_{n}(p)$, then

$$
\begin{aligned}
\lim _{R \rightarrow \infty}\left\{\int_{c-i R}^{c+i R} \mathrm{e}^{p \tau} f_{n}(p) \mathrm{d} p+\int_{\Gamma_{1}} \mathrm{e}^{p \tau} f_{n}(p) \mathrm{d} p\right. \\
\left.+\int_{\Gamma_{2}} \mathrm{e}^{p \tau} f_{n}(p) \mathrm{d} p+\int_{\Gamma 3} \mathrm{e}^{p \tau} f_{n}(p) \mathrm{d} p\right\} \\
=2 \pi i \sum_{m=1}^{\infty} \operatorname{Res}_{p_{n m}}\left[\mathrm{e}^{p \tau} f_{n}(p)\right] .
\end{aligned}
$$

Since $\quad\left|f_{n}(p)\right| \leqslant M|p|^{-\kappa} \quad$ when $\quad|p|>R_{\mathbf{0}}$, where $M, \kappa$ are constants and $\kappa>0$, then, it

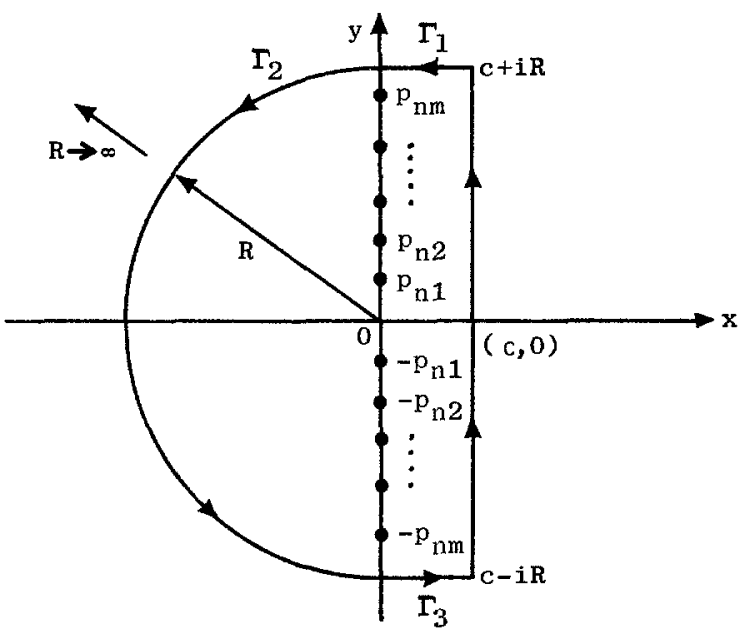

Fig. 1. The path of integration for evaluations of inversion integrals.

can be shown that (i.e., see Scott, 1955)

$$
\begin{aligned}
\lim _{R \rightarrow \infty}\left\{\int_{\Gamma_{1}} \mathrm{e}^{p \tau} f_{n}(p) \mathrm{d} p\right. & +\int_{\Gamma_{2}} \mathrm{e}^{p \tau} f_{n}(p) \mathrm{d} p \\
& \left.+\int_{\Gamma_{3}} \mathrm{e}^{p \tau} f_{n}(p) \mathrm{d} p\right\}=0 .
\end{aligned}
$$

Thus, in view of (31) and (32)

$\frac{1}{2 \pi i} \lim _{R \rightarrow \infty} \int_{c+i R}^{c+i R} f_{n}(p) \mathrm{e}^{p \tau} \mathrm{d} p=\sum_{m=1}^{\infty} \operatorname{Res}_{p_{n m}}\left[\mathrm{e}^{p \tau} f_{n}(p)\right]$.

In (31) and (33) the notation $\operatorname{Res}_{p_{n m}}\left[\mathrm{e}^{p \tau} f_{n}(p)\right]$ denotes the residue of $\mathrm{e}^{p \tau} f_{n}(p)$ at the singularity $p_{n m}$ of $f_{n}(p)$. Since $f_{n}(p)$ are single-valued functions the only singularities of $f_{n}(p)$ are the poles. It can be shown that all the poles of $f_{n}(p)$ are simple and pure imaginary. In general, $\mathrm{e}^{p \tau} f_{n}(p)$ has the fractional form, i.e.,

$$
\mathrm{e}^{p \tau} f_{n}(p)=\frac{h(p)}{g(p)} .
$$

When $\mathrm{e}^{p \tau} f_{n}(p)$ has simple pole at $p=p_{n m}$, $h(p)$ and $g(p)$ satisfy the conditions $g\left(p_{n m}\right)=$ $0, \quad g^{\prime}\left(p_{n m}\right) \neq 0$, and $h\left(p_{n m}\right) \neq 0$ then the residue of $\mathrm{e}^{p \tau} f_{n}(p)$ has the value

$$
\operatorname{Res}_{p_{n m}}\left[\mathrm{e}^{p \tau} f_{n}(p)\right]=\frac{h\left(p_{n m}\right)}{g^{\prime}\left(p_{n m}\right)}
$$


Using (33) and (35) in (28), (29) and (30) after rather lengthy calculations* yields the following expressions for the nondimensional radial and tangential displacements of the shell mid-surface and the velocity potential of the fluid:

$$
\begin{gathered}
\zeta(\varphi, \tau)=\sum_{m=1}^{\infty} \frac{2 R_{0} j_{0}^{\prime 2}\left(\Omega_{0 m}\right) \sin \left(s \Omega_{0 m} \tau\right)}{s\left\{\Omega_{0 m}(2+f) j_{0}^{\prime 2}\left(\Omega_{0 m}\right)+f j_{0}\left(\Omega_{0 m}\right)\left[3 j_{0}^{\prime}\left(\Omega_{0 m}\right)+\Omega_{0 m} j_{0}\left(\Omega_{0 m}\right)\right]\right\}} \\
+\sum_{n=1}^{\infty} \sum_{m=1}^{\infty} \frac{2 R_{n}\left(-s^{2} \Omega_{n m}^{2}+q_{1 n}\right) j_{n}^{\prime 2}\left(\Omega_{n m}\right) \sin \left(s \Omega_{n m} \tau\right) P_{n}(\cos \varphi)}{s d_{n m}\left(-\mathrm{i} s \Omega_{n m}\right)} \\
\psi(\varphi, \tau)=\sum_{n=1}^{\infty} \sum_{m=1}^{\infty} \frac{-2 R_{n} p_{1 n} j_{n}^{\prime 2}\left(\Omega_{n m}\right) \sin \left(s \Omega_{n m} \tau\right) \dot{P}_{n}(\cos \varphi)}{s d_{n m}\left(-i s \Omega_{n m}\right)} \\
\Phi_{1}\left(r_{1}, \varphi, \tau\right)=\sum_{m=1}^{\infty} \frac{2 R_{0} j_{0}^{\prime}\left(\Omega_{0 m}\right) j_{0}\left(\Omega_{0 m} r_{1}\right) \cos \left(s \Omega_{0 m} \tau\right)}{\Omega_{0 m}(2+f) j_{0}^{\prime 2}\left(\Omega_{0 m}\right)+f j_{0}\left(\Omega_{0 m}\right)\left[3 j_{0}^{\prime}\left(\Omega_{0 m}\right)+\Omega_{0 m} j_{0}\left(\Omega_{o m}\right)\right]} \\
\quad+\sum_{n=1}^{\infty} \sum_{m=1}^{\infty} \frac{2 R_{n}\left(-s^{2} \Omega_{n m}^{2}+q_{1 n}\right) j_{n}^{\prime}\left(\Omega_{n m}\right) j_{n}\left(\Omega_{n m} r_{1}\right) \cos \left(s \Omega_{n m} \tau\right) P_{n}(\cos \varphi)}{d_{n m}\left(-i s \Omega_{n m}\right)}
\end{gathered}
$$

where

$$
\begin{aligned}
d_{n m}\left(-\mathrm{i} s \Omega_{n m}\right)=f\left(-s^{2} \Omega_{n m}^{2}+q_{1 n}\right)\left\{\Omega_{n m}\left[j_{n}^{\prime 2}\left(\Omega_{n m}\right)+j_{n}^{2}\left(\Omega_{n m}\right)\right]+j_{n}\left(\Omega_{n m}\right) j_{n}^{\prime}\left(\Omega_{n m}\right)-\frac{\lambda n}{\Omega_{n m}} j_{n}^{2}\left(\Omega_{n m}\right)\right\} \\
+2 f\left(-2 s^{2} \Omega_{n m}^{2}+q_{1 n}\right) j_{n}\left(\Omega_{n m}\right) j_{n}^{\prime}\left(\Omega_{n m}\right)+2 \Omega_{n m}\left(-2 s^{2} \Omega_{n m}^{2}+p_{2 n}+q_{1 n}\right) j_{n}^{\prime 2}\left(\Omega_{n m}\right),
\end{aligned}
$$

$\Omega_{0 m}$ and $\Omega_{n m}$ are the roots of the following frequency equations respectively:

$$
\begin{gathered}
{\left[1+f \frac{j_{0}(\Omega)}{\Omega j_{0}^{\prime}(\Omega)}\right] s^{2} \Omega^{2}-2(1+\nu)=0} \\
{\left[1+f \frac{j_{n}(\Omega)}{\Omega j_{n}^{\prime}(\Omega)}\right] s^{4} \Omega^{4}+\left\{\left[1+f \frac{j_{n}(\Omega)}{\Omega j_{n}^{\prime}(\Omega)}\right]\left(1-\nu-\lambda_{n}\right)\left(1+\alpha^{2}\right)-2(1+\nu)-\alpha^{2}\left[\lambda_{n}{ }^{2}-\lambda_{n}(1-\nu)\right]\right\} s^{2} \Omega^{2}} \\
-(1+\nu)\left\{2\left(1-\nu-\lambda_{n}\right)\left(1+\alpha^{2}\right)+\lambda_{n}\left[1+\nu-\alpha^{2}\left(1-\nu-\lambda_{n}\right)\right]\right\}-\alpha^{2}\left(2-\lambda_{n}\right)\left[\lambda_{n}{ }^{2}-\lambda_{n}(1-\nu)\right]=0 .
\end{gathered}
$$

Equations (39) and (40) were obtained by Engin (1968), for the free vibration analysis of the model under consideration.

From (36) and (37), the response of a closed empty shell subjected to a local radial impulsive load can be easily obtained by setting $f=0$ in (36), (37), (39) and (40); the resulting equations are:

$$
\begin{aligned}
& \zeta(\varphi, \tau)=\frac{R_{0}}{\bar{\Omega}_{0}} \sin \bar{\Omega}_{0} \tau \\
& +\sum_{n=1}^{\infty} \sum_{m=1}^{2} \frac{R_{n}\left(q_{1 n}-\bar{\Omega}_{n m}^{2}\right) \sin \left(\bar{\Omega}_{n m} \tau\right) P_{n}(\cos \varphi)}{\bar{\Omega}_{n m}\left(q_{1 n}+p_{2 n}-2 \bar{\Omega}_{n m}^{2}\right)}
\end{aligned}
$$

$\psi(\varphi, \tau)=\sum_{n=1}^{\infty} \sum_{m=1}^{2} \frac{-R_{n} p_{1 n} \sin \left(\bar{\Omega}_{n m} \tau\right) \dot{P}_{n}(\cos \varphi)}{\bar{\Omega}_{n m}\left(q_{1 n}+p_{2 n}-2 \bar{\Omega}_{n m}^{2}\right)}$

*For some details see Engin (1968). 
where $\bar{\Omega}_{0}=\omega_{0} a / c_{s}$ is the nondimensional breathing mode frequency and its value is directly obtainable from (39); $\bar{\Omega}_{n m}$ are the two distinct roots of the frequency equation (40).

Since the external load was assumed to be of the form $F(\varphi) \delta(\tau)$ the solutions obtained thus far represent the impulse response of the system. In general, a small, but finite length of time elapses during the application of the external load. This means the external pressure should be denoted by $F(\varphi) T(\tau)$, where $F(\varphi)$ has again the same meaning, namely, external load intensity per unit mid-surface area of the shell, and $T(\tau)$ represents any arbitrary pressure time function one desires to choose. Since the analysis is linear the response of the fluid-shell system to an external load $F(\varphi) T(\tau)$ is obtained by means of Convolution integral; denoting the resulting expressions by $\tilde{\Phi}_{1}, \tilde{\zeta}$, and $\tilde{\psi}$ we get

$$
\begin{aligned}
\tilde{\Phi}_{1}\left(r_{1}, \varphi, \tau\right) & =\int_{0}^{\tau} \Phi_{1}\left(r_{1}, \varphi, \xi\right) T(\tau-\xi) \mathrm{d} \xi \\
\tilde{\zeta}(\varphi, \tau) & =\int_{0}^{\tau} \zeta(\varphi, \xi) T(\tau-\xi) \mathrm{d} \xi \\
\tilde{\psi}(\varphi, \tau) & =\int_{0}^{\tau} \psi(\varphi, \xi) T(\tau-\xi) \mathrm{d} \xi
\end{aligned}
$$

When the external load has a finite duration the expression, (4), which gives the velocity imparted to the mass center of the system should be modified to

$$
V_{c}=\frac{1}{M} \int_{S} \int_{0}^{t_{1}} F(\varphi) T(t) \mathrm{d} S \mathrm{~d} t
$$

where $t_{1}$ is the time duration of the external load.

\section{NUMERICAL RESULTS AND DISCUSSION}

In order to use the solutions obtained so far, some ideal conditions must be assumed. In the first place, we assume a spherical form of the brain substance enclosed by the inner layer of the skull cap which is approximately spherical. Furthermore, the brain substance is taken to be homogeneous and as it was pointed out by Goldsmith (1966), since the physical properties of the brain resemble those of a fluid and, in particular, the intercranial-fluid shows some resemblance to water, water is chosen to be the fluid occupying the interior space of the spherical shell. The skull cap which is idealized by the thin shell is also taken to be homogeneous and isotropic. Under these assumptions we arrive at the following data:

$$
\left.\begin{array}{rl}
\rho_{s} & =0.0772 \mathrm{lbm} / \mathrm{in}^{3} \\
E & =2 \times 10^{6} \mathrm{lbf} / \mathrm{in}^{2} \\
\nu & =0.25 \\
a & =3 \mathrm{in} . \\
h & =0.15 \mathrm{in} . \\
\rho_{0} & =0.0362 \mathrm{lbm} / \mathrm{in} . \\
c & =57,100 \mathrm{in} . / \mathrm{sec}
\end{array}\right\} \quad \text { For the shell }
$$

From the shell data we note that $a / h$ is within the justifiable thin shell theory limits; also the calculated value for $c_{s}$ is $103,280 \mathrm{in} / \mathrm{sec}$ which is in close agreement with the wave speed of $106,000 \mathrm{in} . / \mathrm{sec}$ through the skull mentioned in Goldsmith's paper. The axisymmetric external impulsive load is considered to be applied on the shell with a constant magnitude of $546.5 \mathrm{lbf} / \mathrm{in}^{2}$ on a polar cap of $15^{\circ}$ angle. Thus, the addition of $F_{0}=546.5$ $\mathrm{lbf} / \mathrm{in}^{2}$ and $\phi_{0}=15^{\circ}$ to the above completes the necessary data.

In the determination of poles ( $\mp$ is $\Omega_{n m}$ ) and corresponding residues the spherical Bessel functions play an important role. Unavailability of the spherical Bessel function subroutine compelled us to write a subroutine by using a recursion formula and the series representation of the spherical Bessel function. Since for the small arguments the recursion formula gives unstable results for the values of the spherical Bessel function, the series representation which exhibits strong convergence for the small arguments is substituted. For each order, $n$, of the spherical Bessel function and its first derivative an argument was determined for which both recursion and the series representation 
gave the same result to within the desired accuracy. Thus, the subroutine was designed to call for the series representation when the arguments are less than a particular number and the recursion formula otherwise.

The nondimensional excess pressure, $p_{1}$, is equal to $-\partial \Phi_{1} / \partial \tau$ and it is obtainable directly from (38). If we consider one to one correspondence between the natural frequencies and the modes of the system, for $n=0, \ldots, 20$ and $m=1, \ldots, 20$ there are 420 modes which were partly computed as residues to get the shell mid-surface displacement components $\zeta, \psi$, and the excess pressure $p_{1}$. For $\zeta$ and $\psi$ less than half of these modes give sufficient convergence; but for the fluid pressure it was necessary to use all of the modes. Having determined the mid-surface displacement components $\zeta$ and $\psi$, we obtain the mid-surface strains in the case of axisymmetric torsionless motion of the shell from the following expressions:

$\epsilon_{\varphi}=\frac{\partial \psi}{\partial \varphi}+\zeta \quad \kappa_{\varphi}=\frac{1}{a}\left(-\frac{\partial^{2} \zeta}{\partial \varphi^{2}}+\frac{\partial \psi}{\partial \varphi}\right)$

$\epsilon_{\theta}=\psi \cot \varphi+\zeta \quad \kappa_{\theta}=\frac{\cot \varphi}{a}\left(-\frac{\partial \zeta}{\partial \varphi}+\psi\right)$.

In (46) $\epsilon_{\varphi}$ and $\epsilon_{\theta}$ are the tangential strains, whereas $\kappa_{\varphi}$ and $\kappa_{\theta}$ can be viewed as the variations of the curvature of the mid-surface of the shell during deformation. It can be shown (i.e. see Novozhilov, 1964) that the $z$-surface* strains are related to those of the mid-surface in the following manner:

$$
\begin{aligned}
\epsilon_{\varphi}{ }^{(z)} & =\frac{1}{1+z / a}\left(\epsilon_{\varphi}+z \kappa_{\varphi}\right), \\
\epsilon_{\theta}{ }^{(z)} & =\frac{1}{1+z / a}\left(\epsilon_{\theta}+z \kappa_{\theta}\right) .
\end{aligned}
$$

By Hooke's law and the hypothesis of Kirchoff, one has for a homogeneous and isotropic shell the following stress-strain relations.

$$
\begin{aligned}
& \sigma_{\varphi}^{(z)}=\frac{E}{1-\nu^{2}}\left(\epsilon_{\varphi}^{(z)}+\nu \epsilon_{\theta}^{(z)}\right), \\
& \sigma_{\theta}^{(z)}=\frac{E}{1-\nu^{2}}\left(\epsilon_{\theta}^{(z)}+\nu \epsilon_{\varphi}^{(z)}\right),
\end{aligned}
$$

where $E$ is Young's modulus and $\nu$ is Poisson's ratio. Thus, the stress distribution in the shell is found at any given time by substituting (36) and (37) into (46) (47) and (48).

On Figs. 2-9 the numbers enclosed by small circles designate the multiples of the time increment. The time increment chosen represents $\frac{1}{10}$ of the calculated time which the stress wave on the shell takes to arrive at the opposite pole. Thus, (1) refers to actual time of $t=9.125 \times 10^{-6} \mathrm{sec}$ or nondimensional time $\tau=0.3141$ and (10) refers to actual time of $t=9.125 \times 10^{-5}$ or nondimensional time $\tau=3 \cdot 141$. On Figs. $2-5$ the circle of radius $r_{1}$ represents the zero magnitude of stress; the outward direction is used for the compressive and the inward direction for the tensile stresses. We can make the following remarks based on a close study of the numerical results and the graphs shown on Figs. 2-9:

(1) The magnitudes of stress components $\sigma_{\varphi}$ and $\sigma_{\theta}$ acting on the mid-surface of the shell $(z=0)$ in the fluid-filled case are considerably less than those of the in vacuo case. The obvious reason for this is the presence of the high density fluid which absorbs a large portion of the initial energy input from the shell material whose modulus of elasticity is quite low.

(2) In the fluid-filled case the maximum compressional stress on the mid-surface of the shell at the opposite pole occurs at a later time, namely, at the time (14) whereas in the in vacuo case this time is (10).

(3) Time (II) corresponds approximately to the arrival of the compressional fluid pulse at the opposite pole. The magnitude of the tensile stress on the mid-surface of the shell at this time is higher than the magnitude of the compressive stress at time (10). This

\footnotetext{
*Any surface which is equidistant from the mid-surface is called a $z$-surface; for the mid-surface $z=0$. The possible values of $z$ are $-h / 2 \leqslant z \leqslant h / 2$, where $h$ is the shell thickness.
} 


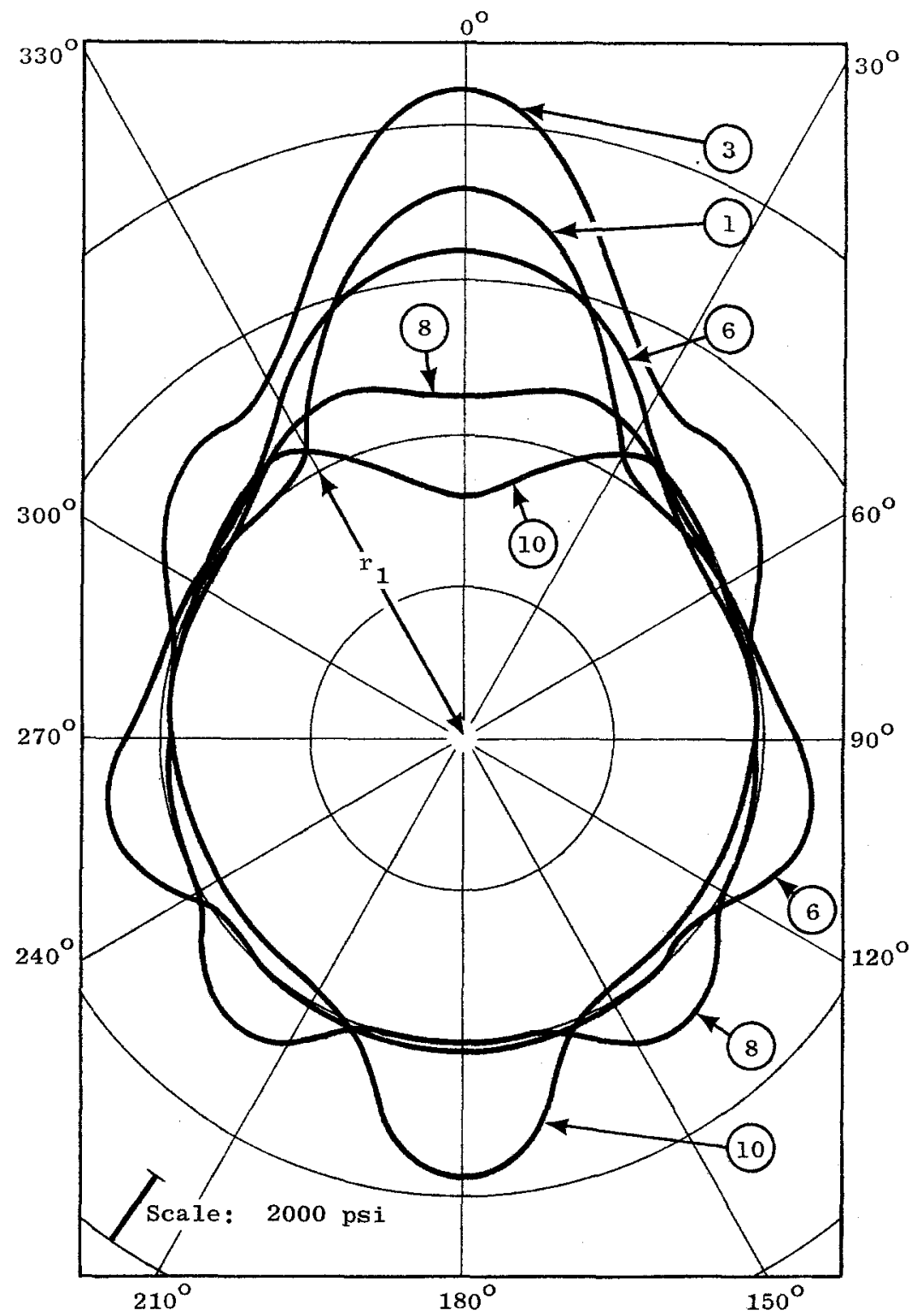

Fig. 2. Normal stress in the $\varphi$-direction as a function of the polar angle, $\varphi$, at various times (in vacuo case), $z=0$. 


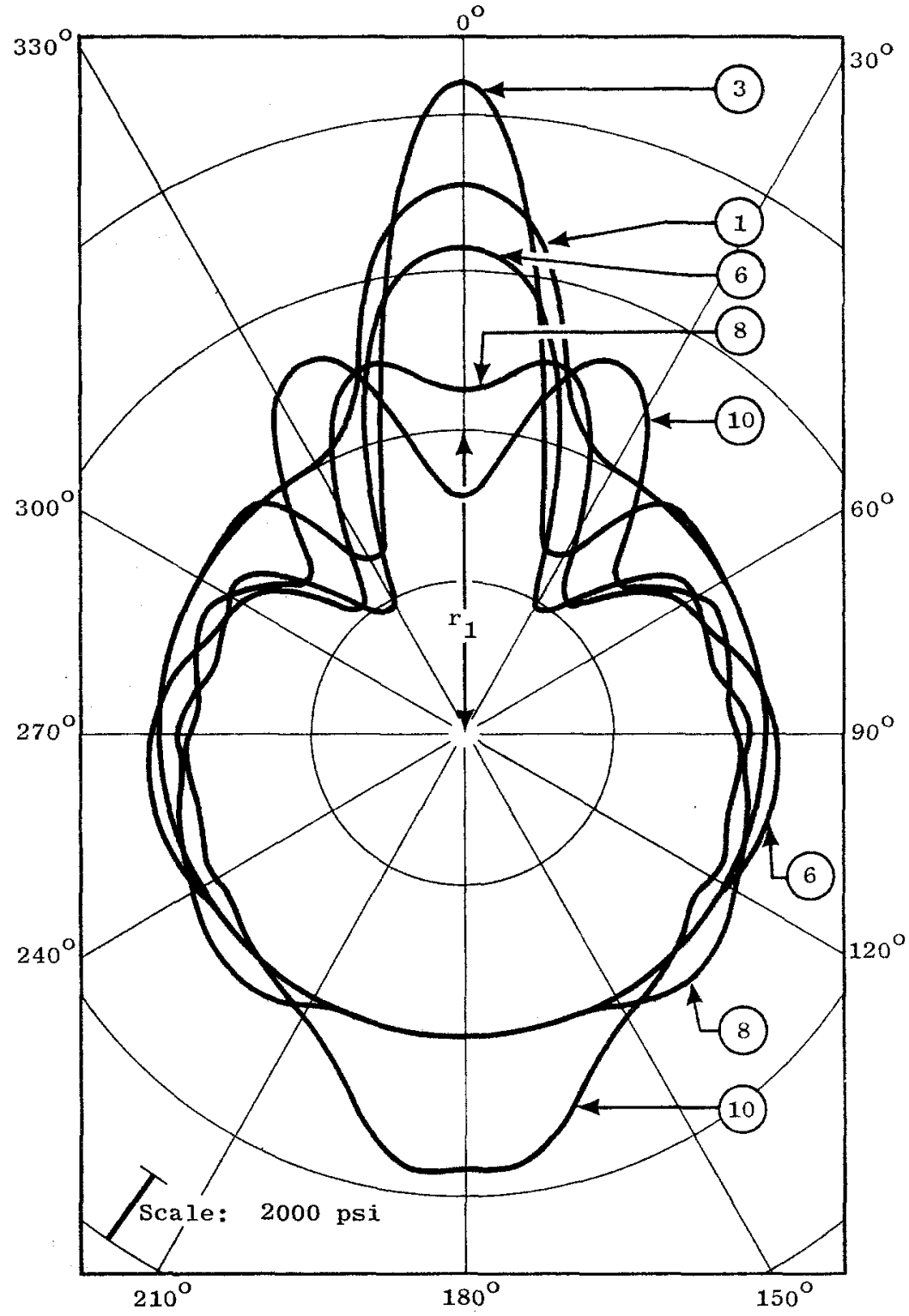

Fig. 3. Normal stress in the $\theta$-direction as a function of the polar angle, $\varphi$, at various times (in vacuo case), $z=0$. 


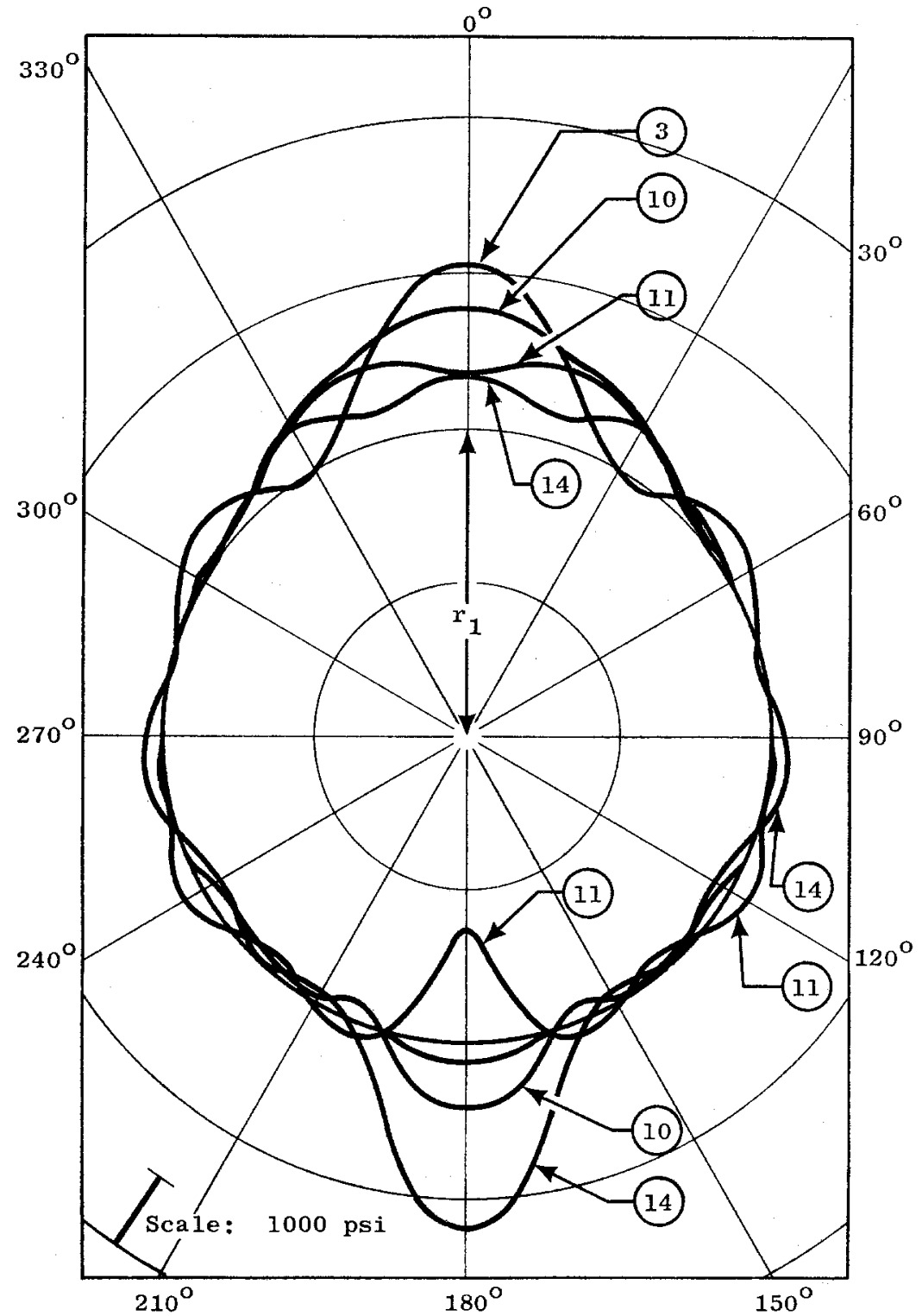

Fig. 4. Normal stress in the $\varphi$-direction as a function of the polar angle, $\varphi$, at various times (fluid-filled case), $z=0$. 


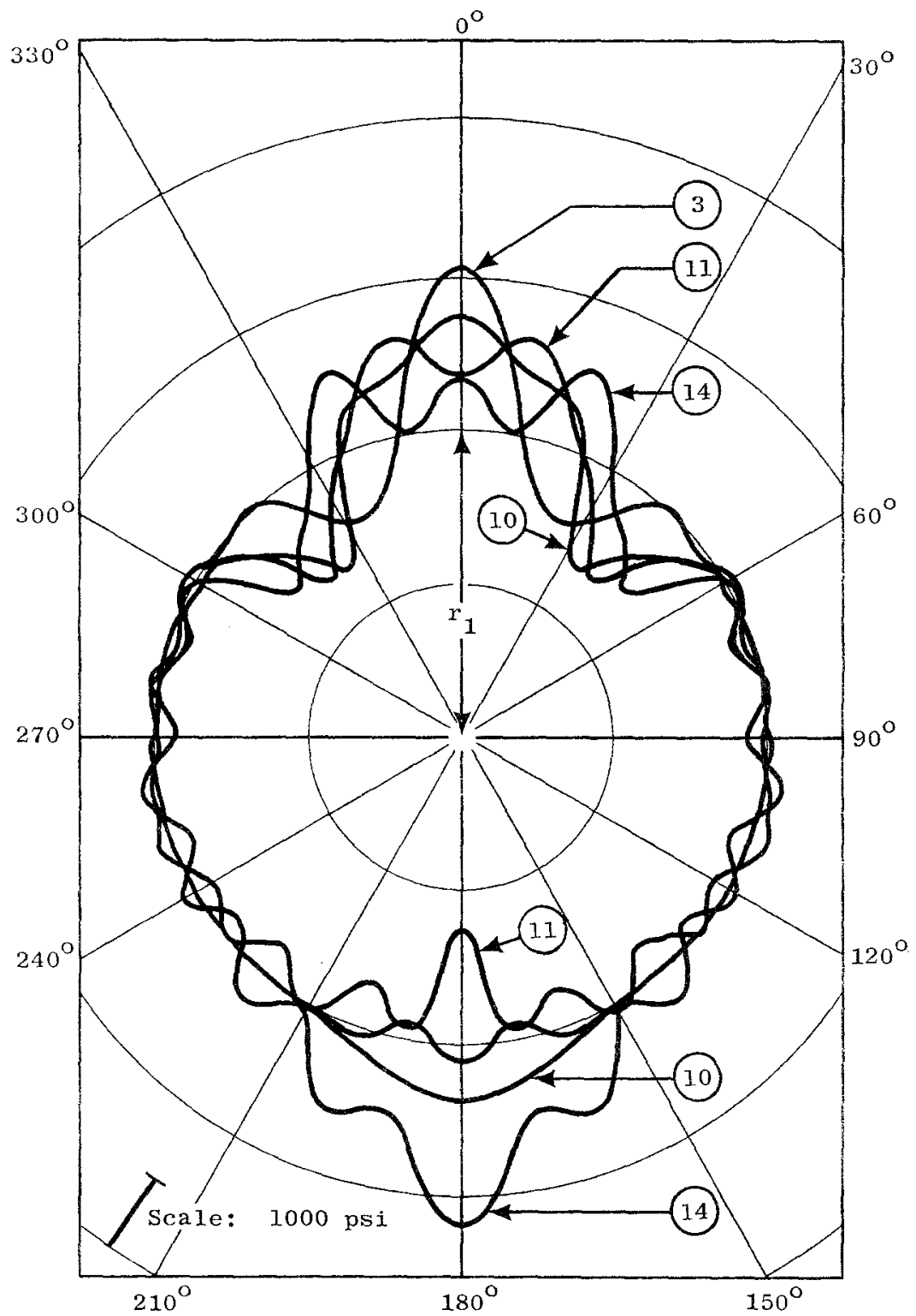

Fig. 5. Normal stress in the $\theta$-direction as a function of the polar angle, $\varphi$, at various times (fluid-filled case), $z=0$. 
increase is caused by the reflection of the compressional fluid pulse at the opposite pole.

(4) Figure 6 shows for both the fluid-filled and in vacuo cases, the occurrence of the maximum inward and outward radial shell displacements at the pole where the impulsive load is applied. Figure 7 is a plot of the stress distribution throughout the shell thickness at those times when the radial displacements are maximal for each of the two cases.

(5) In Fig. 8, the nondimensional excess pressure is plotted as a function of the nondimensional radial distance. From this plot we can see the propagation of the compressional pulse toward the center of the fluid. There is a decrease in magnitude of the pulse as it progresses toward the center of the sphere. We keep in mind that this pulse represents a rather complex superposition of pulses generated on, and reflected, with varying strengths from the shell surface as the compressional disturbance on the shell propagates from the pole of loading toward the opposite pole.

(6) Figure 9 shows a similar plot, but the radial distance is taken from one pole to the other. The purpose of this plot is to show the occurrence, location, and magnitude of the maximum negative excess pressure. The magnitude of this negative excess pressure is indeed higher than the magnitude of the maximum positive excess pressure.

\section{CONCLUSIONS}

In view of Figs. 4, 5 and 7 we can state that the possible locations on the skull susceptible to severe damage are:

(a) The pole $\left(\varphi=0^{\circ}\right)$ where the impulsive load is applied; here the fracture starts at the inner surface $(z=-h / 2)$ of the skull which is in the state of inbending.

(b) The neighborhood of $\varphi=35^{\circ}$ where tensile stresses develop repeatedly; this is a

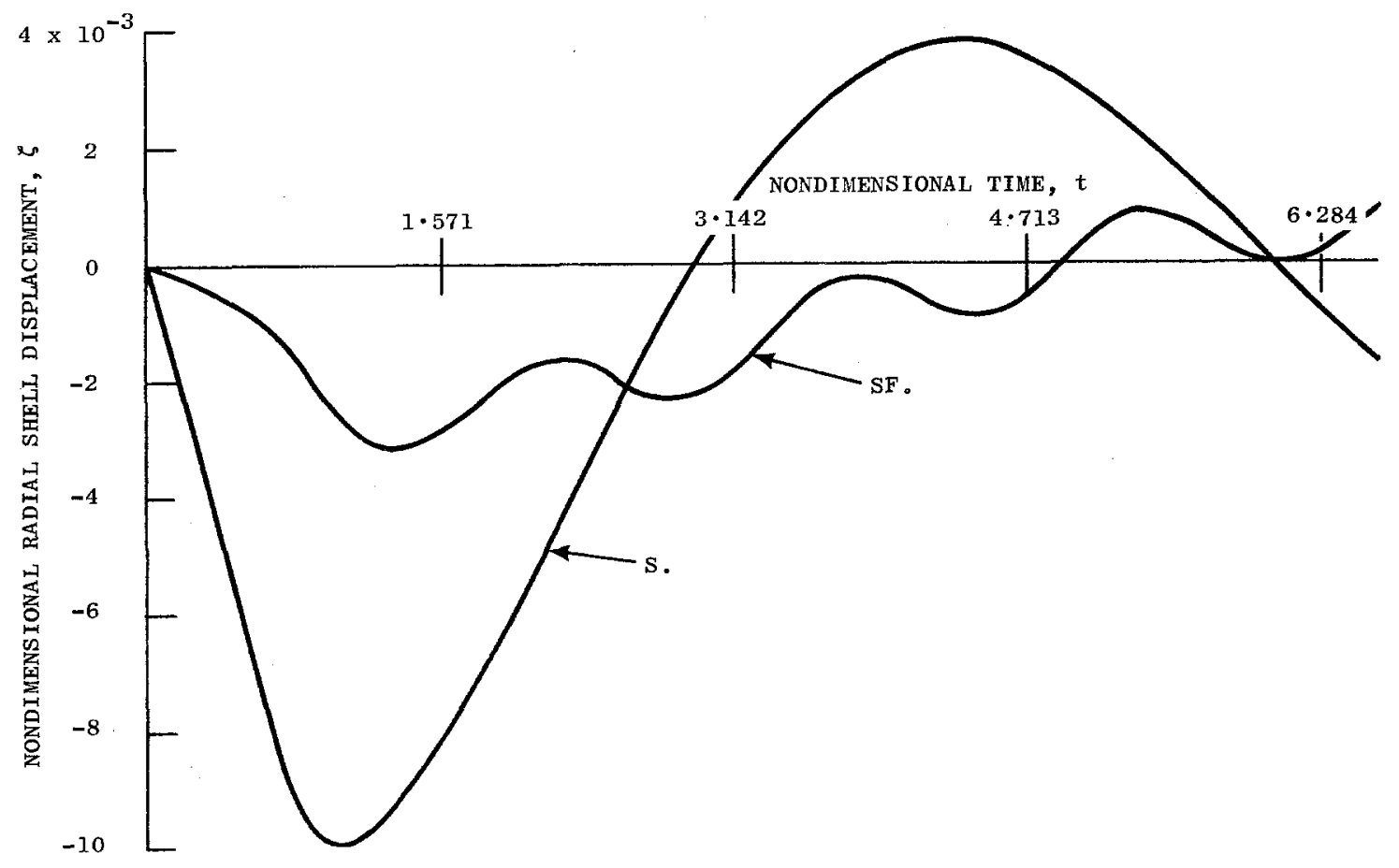

Fig. 6. Nondimensional radial shell displacement vs. time, $\phi=0$. 

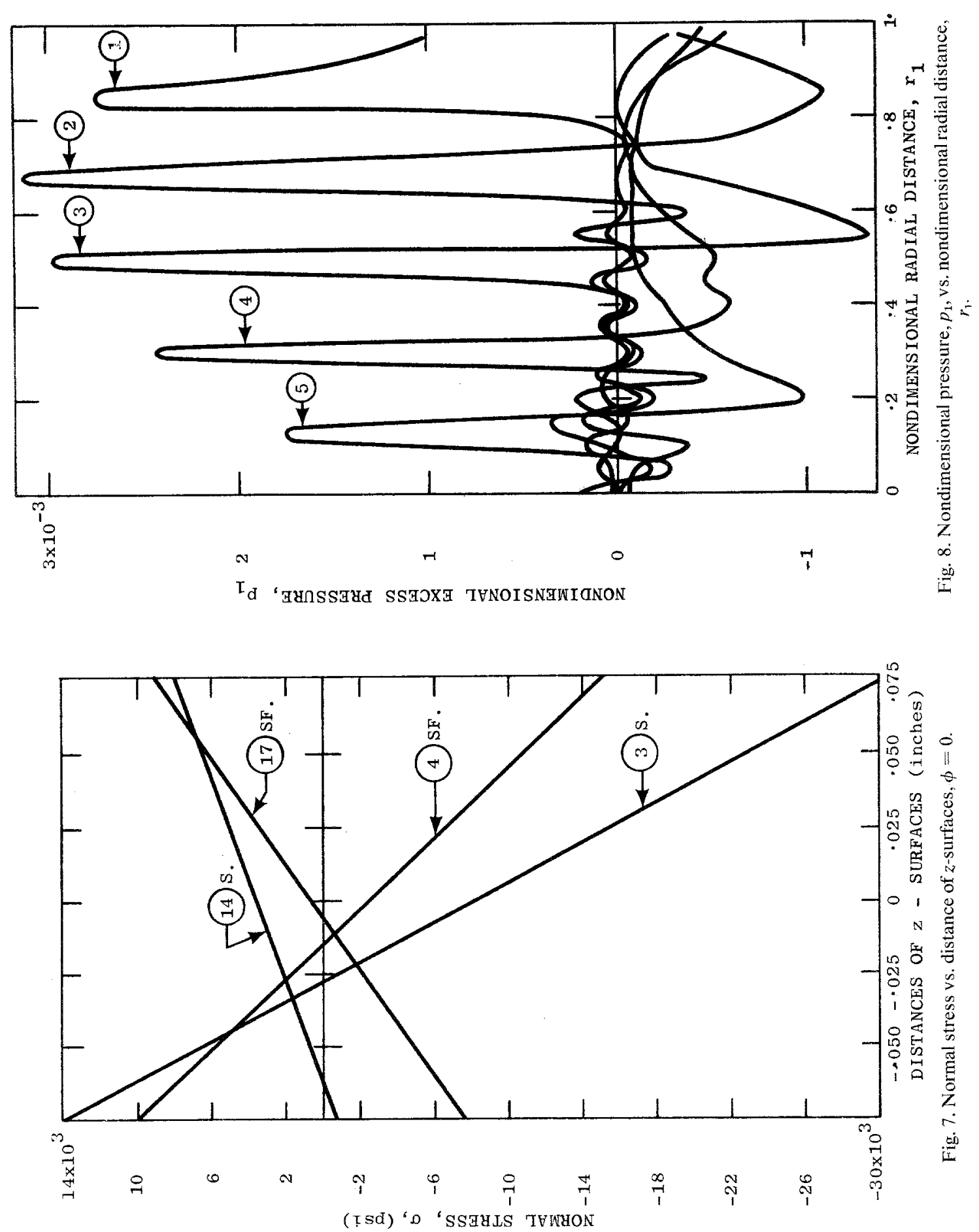


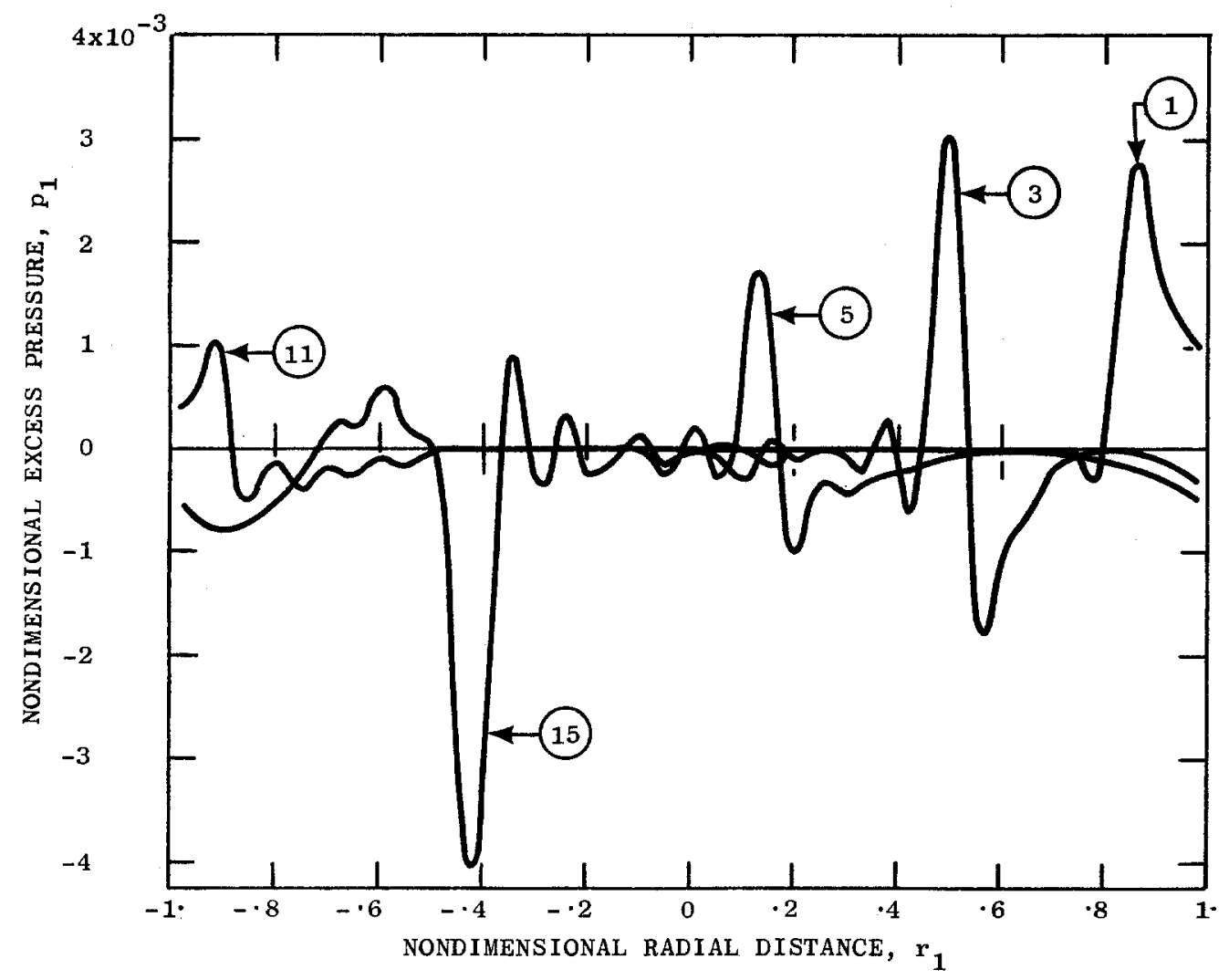

Fig. 9. Nondimensional pressure, $p_{1}$, vs. nondimensional radial distance, $r_{1}$.

circular region around the pole and it is in the state of outbending.

(c) the opposite pole $\left(\varphi=180^{\circ}\right)$ where high values of tensile stress are generated after the reflection of both the solid and the fluid waves one after the other.

On the basis of experiments conducted by E. S. Gurdjian, H. R. Lissner and J. E. Webster the locations (a) and (b) were discussed by F. G. Evans (1957) in the fifth chapter of his book titled "Stress and Strains in Bones."

If we seek the brain damage to occur at the points of rarefaction of the fluid, then Fig. 9 indicates that this situation arises at time (3) and location $r_{1}=0.55$ and much more severely at time (15) and $r_{1}=-0.43$. This fact clearly establishes the difference between the present and rigid shell analysis where it was inferred that maximal brain damage occurs at the center. The locations of rarefaction of the fluid are quite significant for the analytical confirmation of the cavitation theory of brain damage. Finally, the occurrence of a reduced pressure in the region close to the opposite pole sheds some light to the explanation of the so-called contre-coup phenomenon.

Acknowledgements - This paper is based upon a dissertation submitted by the writer to the Graduate School in The University of Michigan, in partial fulfillment of the requirements for the Ph.D. degree. The supervision of the work was provided by Professors Y. K. Liu and R. D. Low. Financial aid was supplied by the Biomechanics Department of the Highway Safety Research Institute of The University of Michigan under Contract No. 43-67-1136 sponsored by the National Institute for Neurological Diseases and Blindness. 


\section{REFERENCES}

Anzelius, A. (1943) The effect of an impact on a spherical liquid mass. Acta. path. microbiol. scand. Suppl. 48.

Engin, A. E. (1968) The axisymmetric response of a fluid-filled spherical shell. A dissertation submitted to the Graduate School in The University of Michigan.

Evans, F. G. (1957) Stress and Strain in Bones. pp. 34-53. Thomas, Springfield, Ill.

Goldsmith, W. (1966) The physical processes producing head injury. In Proceedings of the Head Injury Conference. pp. 350-382. Lippincott.

Guittinger, W. (1950) Der Stosseffekt auf eine Flüssigkeitskugel als Grundlage einer Physikalischen Theorie der Entstehung von Gehirnverletzungen. $Z$. Naturf. A5, 622-628

Novozhilov, V. V. (1964) Thin Shell Theory. 2nd Edn. Noordhoff, Groninger, Holland.

Scott, E. J. (1955) Transform Calculus with an Introduction to Complex Variables. pp. 56-60. Harper and Brothers, New York.

\section{NOMENCLATURE}

$E$ Young's modulus

$F_{e}$ external force distribution on the shell

$H(t)$ heavyside unit step function

$M$ total mass of the system, $m_{0}+m_{s}$

$P_{n}(\cos \phi) \quad$ Legendre polynomials of the first kind

$P_{n}^{\prime}(\cos \phi)$ associated Legendre polynomials of the first kind and first order

$S$ mid-surface of the shell

$V_{c}$ velocity imparted to the mass center

$\Phi$ velocity potential for the fluid

$\Phi_{1}$ nondimensional velocity potential for the fluid, $\Phi / a c_{s}$

$\Omega$ nondimensional frequency, $\omega a / c$

$\bar{\Omega}$ nondimensional frequency, $\omega a / c_{s}$

a radius of spherical shell $c$ compressional wave speed in the fluid

$c_{s}$ apparent wave speed in the shell, $\left[E / \rho_{s}(1-\nu)^{2}\right]^{1 / 2}$

$f$ shell-fluid parameter, $\rho_{0} a / \rho_{s} h$

$h$ shell thickness

$j_{n}(z) \quad$ spherical Bessel function, $(\pi / 2 z)^{1 / 2} j_{n+1 / 2}^{(z)}$

$k$ wave number, $\omega / c$

$m_{0}, m_{s}$ masses of fluid and shell respectively

$p$ complex variable for the Laplace transform or pressure

$p_{a} \quad$ fluid pressure on the surface of the shell

$p_{1}$ nondimensional pressure, $p / \rho_{0} c_{s}{ }^{2}$

$r, \theta, \varphi$ spherical coordinates

$r_{1}$ nondimensional radius, $r / a$

$s$ speed ratio, $c / c_{s}$

$t$ time

u meridional displacement with respect to center of mass of the system

$w$ radial displacement with respect to center of mass of the system

$z$ distance from the mid-surface, or complex variable

$\alpha^{2} \quad$ thickness parameter, $h^{2} / 12 a^{2}$

$\delta(t)$ Dirac delta function

$\epsilon_{\varphi}, \epsilon_{\theta}$ mid-surface normal strains

$\epsilon_{\varphi}{ }^{(z)}, \epsilon_{\theta}{ }^{(z)} \quad z$-surface normal strains

$\zeta$ nondimensional radial displacement, $w / a$

$\psi$ nondimensional meridional displacement, $u / a$

$\kappa_{\varphi}, \kappa_{\theta} \quad$ mid-surface curvatures

$\lambda_{n} n(n+1)$

$\nu$ Poisson's ratio

$\rho_{0}, \rho_{s}$ mass density of fluid and shell respectively

$\sigma_{\varphi}, \sigma_{\theta}$ normal stresses for the mid-surface

$\sigma_{\varphi}^{(z)}, \sigma_{\theta}^{(z)}$ normal stresses for the $z$-surface

$\tau$ nondimensional time, $c t / a$

$\omega$ angular frequency. 